\title{
Preparation of controlled particulate mixtures with glass beads of different sizes
}

\author{
Ricardo Dias, José A. Teixeira, Manuel Mota*, Alexander Yelshin \\ Centro de Eng. Biológica, IBQF, University of Minho, Campus de Gualtar, Braga 4710-057, Portugal
}

Accepted 30 July 2003

\begin{abstract}
A method of mixing/preparing binary and ternary mixtures of glass beads was developed using a viscous solution of glycerol in water. After mixing, the mixture was transferred to a prismatic vessel and glycerol was washed out. The different sized beads were differently coloured and digital pictures taken from each face were automatically treated by image analysis to determine the coloured fraction present in each face. Statistical analysis showed that no significant deviation existed in the colour distribution of each of the four faces. A chi-square test showed that a uniform distribution could be accepted for the beads, no segregation of bead size nearby the edges was observed and that no wall effect was present. The two-dimensional picture obtained by image analysis was converted to the corresponding three-dimensional distribution, from which the expected bed porosity was inferred. The porosity previously estimated was compared with the experimental porosity determined by gravimetry directly on the bed. No significant deviations were found, thereby proving that the mixing method developed was reliable. Hundreds of experiments were done showing a very high reproducibility. The developed method was further used for studies on mixing of binary and ternary mixtures. In certain conditions (mixtures enriched with large size particles and having a significant difference in size) a segregation layering effect took place and the bottom layer presented a composition corresponding to the packing with the smallest porosity possible. (c) 2003 Elsevier B.V. All rights reserved.
\end{abstract}

Keywords: Particle mixtures; Packed beds; Spherical particles; Modelling; Porosity

\section{Introduction}

Mixed beds of particles have a wide application in industry and sciences. Granular beds and, in particular, spheres packing of different sizes display a wide range of values of porosity $(\varepsilon)$, pore size $\left(d_{e}\right)$ or particle size $\left(d_{p}\right)$ and pore channel tortuosity [1-5]. For this reason, the choice of a correct packing bed model as well as of a procedure of particles mixing

\footnotetext{
* Corresponding author. Tel.: +351-253-604400; fax: +351-253-678986.

E-mail address: mmota@deb.uminho.pt (M. Mota).
}

and packing remain an important subject of theoretical and experimental investigations [6-11]. Study of the above mentioned parameters is difficult whenever the pore topology is unknown or difficult to control. This is the case of natural porous media, as well as of filtration media made of irregular particles such as kieselguhr. A way to overcome this setback is to construct spherical packed beds with a well-defined topology, which may serve as experimental models. This may be accomplished if the mixture is controlled by using a reproducible mixing method.

The purpose of this work is, in the first place, to elaborate an improved and reproducible method of 


\begin{tabular}{|ll|}
\hline Nomenclature \\
$d_{e}$ & pore size \\
$d_{i}$ & particle size of $i$ th fraction in the mixture \\
$D_{p}$ & particle size \\
$d_{\mathrm{pi}}$ & particle size of $i$ th fraction \\
$N_{c}$ & coordination number \\
$S_{0}$ & specific particle area \\
$x_{i}$ & volume fraction of particles of $i$ th fraction \\
$\varepsilon$ & porosity \\
\hline
\end{tabular}

mixing/packing spherical glass beads for further investigation of the porosity-tortuosity relationship of binary and ternary mixtures. Finally, we shall attempt to analyse the range of possible models based on the overall averaging procedure and to model segregated mixtures.

\subsection{Porosity variation range}

\subsubsection{Mono-sized bed}

Due to the random nature of mixed beds as well as to the different preparation methods applied, there is quite a wide variation even in the case of mono-sized beds $[1,12-15]$.

Depending on packing conditions it is possible to obtain some regular packing of spheres as well as a randomised packing [16,17]. The regular packing related with a fixed coordination number $N_{c}$ corresponds to the number of contacts of each sphere in the bed with the neighbouring spheres. For instance, coordination numbers and porosity of a regular mono-sized packing can be found in the work [16], from where it follows, according to [18], that within the class of mono-sized beds the porosity stays in the range $0.2595-0.4764$ and depends on the particle arrangement.

\subsubsection{Random particle packing}

The random mono-size particle packing also displays a wide porosity range [16]. For instance, diffusion through mono-dispersed silica spherical particles packing was investigated by [19]. Six beds were formed from mono-sized particles of diameter from 52 up to $305 \mathrm{~nm}$. The same procedure of packing was used; nevertheless, the average porosity was in the range $0.331-0.381$. In the work [20] for mono-sized silica spheres of diameter $132 \mathrm{~nm}$ a porosity around 0.36 and 0.39 was obtained whereas for a particle size of $300 \mathrm{~nm}, \varepsilon \approx 0.3754$ and for $402 \mathrm{~nm}, \varepsilon \approx 0.42$, respectively. Hence, packing density depends on the nature of inter-particles interaction. Furthermore, the structure depends on the packing boundary condition [21].

Transition from dense to loose packing results in decreasing the average number of the contact points and with increasing porosity may lead to unstable structure when porosity $\varepsilon>0.5-0.6$. Moreover, in the absence of interaction forces (Van der Waals, sticking, etc.) contacts may be impossible between spheres. For example, in the work [22] a new method of building up the loose packing was developed. Polystyrene beads were pre-mixed with different amounts of sugar and packed into a column. After bed treating by $\mathrm{CCl}_{4}$, polystyrene beads stuck together and formed a rigid skeleton. Finally, sugar was washed out from the bed.

\subsubsection{Multi-component mixtures}

For mixtures of particles of different size the overall porosity also varies in a certain range dependent on the particle size ratio, particle composition, method of mixing/packing and can be reduced down up to $\sim 0.1-0.2$ [8-10,23-26].

In some special cases a particle size ratio of different fractions is close to a situation in which smaller particles can be inserted exactly into the free space between larger ones, thereby creating an extremely dense packing, for example in the case of ternary mixtures [27,28].

Experimental and theoretical observations show that binary and ternary mixtures of particles of different size are able to fulfil a wide span of conditions for granular packing beds-porosity, permeability, pore size, specific surface area $\left(S_{0}\right)$, etc. Moreover, by increasing the number of discrete particle size fractions, transition to a continuous particle size distribution $[9,10]$ is observed and makes difficult the predictability and control of porous medium properties [24,29].

Nevertheless, when components in the multimodal continuous particle size distribution can be separated (approximated) in fractions, it is possible to apply a discrete mixture model $[8,26,27]$. This approach facilitates the analysis of the influence of each particle fraction on the overall mixture porosity. 


\subsection{Pore size}

In general the average particle size $d_{\mathrm{av}}$ in a mixture of particles of $n$ different size can be represented as [5]

$\frac{1}{d_{\mathrm{av}}}=\sum_{1}^{n} \frac{x_{i}}{d_{\mathrm{pi}}}$

where $x_{i}$ and $d_{\mathrm{pi}}$ are the volume fraction and particle size of $i$ th fraction, respectively. As particle and pore size of spheres packing and specific area are related with particle size, the average pore size in the first approach is $d_{\mathrm{eav}}=2 d_{\mathrm{av}} \varepsilon / 3(1-\varepsilon)$ and $S_{0}=6 / d_{\mathrm{av}}$.

Two comments can be made to Eq. (1): (i) The pore diameter depends on porosity and particle size whereas both depend on packing fractional content; (ii) binary and ternary particle mixtures are able to generate porous media displaying a wide range of average pore size.

\subsection{Tortuosity}

Tortuosity is usually defined as an average ratio of the pore length to the bed thickness. In particular, the tortuosity characterises the ratio of the distance a substance (macromolecule, micro-particles, etc.) has to cover in a porous medium to the medium bed thickness. Tortuosity is associated with the flow and mass transfer characteristics such as permeability, diffusivity, effectiveness, etc. [1,2,30-38].

Theoretical and practical investigations show that the tortuosity of a granular bed is defined by the fractional content, porosity, and particle shape. Therefore its value may cover a wide range [8,11,26,27,39].

Besides the fractional content and particle size ratio between fractions, the way of mixing particles and the packing method can also alter granular bed or layer structure $[8,12,14,15,20,40,41]$. Depending on the size ratio, two packing mechanisms are involved-a filling mechanism and an occupation mechanism [23]. The packing procedure may give rise either to an equilibrium packing density or to segregation effects of different nature [42-44].

\subsection{Models of mixed bed of particles of different size}

The transition from uniform particle packing to the multi-component mixed bed of different particle size makes the porous medium properties more complicated. A granular system may be in a large number of different microscopic states at fixed macroscopic densities [45] and, hence, at the same porosity, the granular bed may exhibit, for example, different permeability, tortuosity, etc. For example, the functional dependence of the binary mixture porosity versus particle volume fraction has an identical porosity value for two different particle volume fractions. An even more complex dependence may be found in ternary mixtures.

Nevertheless, binary mixtures are still the best modelling system for the investigation of transport phenomena in porous media. Ternary mixtures are more complex to deal with. Some authors [46] observed the following fact for ternary mixtures-the mixture minimum porosity zone is close to the binary mixture axis representing the largest and smallest particle size fractions. Similar modelling results may be seen, for instance, at [23]. Other authors [27] showed that by controlling the way of filling fixed binary mixture's void space by the fine particle fraction a very dense packing can be achieved.

The porosity $\varepsilon$ of mixed particle beds has been described in many models of binary and ternary mixtures of spheres $[23,46,47]$ as well as of non-spherical particles [15,48-51]. The obtained model relationships adopted for the porosity description are complicated for investigation and modelling of the permeability and the tortuosity behaviour in mixed beds.

Models are often built on the basis of averaged parameters when physical characteristics are defined with respect to the overall space occupied by the mixture. In some cases however the mixture corresponds to a segregated layered system and therefore some averaged parameters might be quite far from real values of, for example, tortuosity [52].

\section{Experimental part}

\subsection{Choosing mixing/packing procedures}

To show the validity of packing procedures for the mixture preparation a brief review is outlined below. Usually, a process of packing formation includes two major steps-mixing of components and packing when the mixture is placed into a column or vessel. 
In a published work [40] the densification process experienced by a bed of frictional spheres of diameter $d$ in a rectangular vessel whose floor was subjected to high frequency and low amplitude sinusoidal oscillations was modelled using discrete element simulations. Beginning with an initial random assembly resulting from gravity deposition, the floor motion activated the system so as to induce the formation of a distinct microstructure in the absence of a mean flow field. This was accompanied by an increase in the coordination number and bulk solids fraction whose evolution was strongly dependent on the magnitude of the acceleration amplitude. The method drawback is the segregation effect when particles have different sizes.

A wet method of packing was applied in [12]. To avoid air bubbles in the packing during the permeability measurements the empty tube was first filled with water before adding particles. To minimise the formation of heterogeneous regions in the packing, spherical particles were added in five batches, each formed by mixing the appropriate amounts. Particles for each batch were added in small spoonfuls and, after allowing their settling in the bed, the tube was softly tapped on the side to promote close packing. This method is limited for large particle size ratio when differences in settling velocities are significant.

Two methods of filling industrial catalyst grade packing with fines were described in [14], portion mixing and whole-bed packing procedures. Methods included loading of the catalyst to the reactor and gently tapping or vibrating the reactor until the catalyst bed settled down. These methods are applicable for particles with large size ratios when fines are able to migrate to the void space.

For non-spherical mixtures, other authors [15] used two packing methods-loose and dense random packing. The main experimental procedure is given as follows. The particles required for a given mixture were first weighed, pre-mixed manually and poured into a cylindrical container through a funnel. They were then shaken vigorously in a circular, end-over-end fashion. The cylinder was tipped horizontally, slowly rotated about its axis, and gradually returned to the vertical position to produce loose random packing. Gentle vertical tapping was then applied until no volume variation could be observed to produce dense random packing. A similar procedure was applied in [8]. The proce- dure needs careful standardisation to ensure packing homogeneity.

A procedure for homogeneously mixing of two different sizes of particles in a horizontally rotating cylinder was presented in [41]. The authors found that different cylinder rotation rates produce inverted segregation patterns. A radial core of small particles is formed at low rotation rates and a core of large particles is formed at high rotation rates. A homogeneous mixture can be obtained by alternating rotation rates at which particles occupy corresponding radial positions. In turn, in [20], a mixture of two mono-disperse sphere fractions of different sizes was made by blending the desired weight proportions in an agitated flask. After $30 \mathrm{~min}$ agitation via a magnetic stirrer, the binary mixture was organised in pellets.

The mixing of glass beads by rotating a cylindrical vessel about its axis and rocking it vertically was investigated at [13]. The authors detected that the mixing process is enhanced to a greater extent when the rocking frequency is different from the rotational frequency to avoid resonance. The best result was achieved for a rocking frequency (number of rocking cycles per revolution) greater than one.

It must be mentioned that, even when a perfect particle mixture is poured into a vessel, clustering and segregation effects may be observed, especially if the particle size ratio is significant. In an ideal system particles should undergo uniform falling flux onto the bed surface [53]. In order to preserve the desired particle composition it would be desirable to fix the particles, for example, by adhesion.

Authors [54] studied effects of changing the binder viscosity in rotating drum granulation of a narrow size fraction of irregularly shaped sand. Silicone fluids, with a viscosity range between 20 and $500 \mathrm{mPa}$ s, were used as binders. They found that the viscosity of the binder affected both the rate and the mechanism of aggregate enlargement. The growth rate increased with the increase in binder viscosity up to a maximum at a viscosity of about $100 \mathrm{mPa}$. Enlargement occurred by a layering mechanism. With binders of viscosity higher than $100 \mathrm{mPas}$, layering was not observed and growth was found to be due to coalescence.

In the experiments presented in this work, we tested a similar method of mixture preparation using a water solution of glycerol. Helicoidal impellers are commonly used in the mixing of soap and paper pastes. 
Dry solids of different dimension, like calcium carbonate, cement, sand and cellulose fibbers are mixed with multi-helicoidal impellers in the tile adhesive industry. Application of glycerol for particles adhesion on the later stage of mixture packing gives the possibility of removing adhesive from a column with a minimum cost. Preliminary experiments show that the optimal solution for mixing is the $90 \%$ solution of glycerol in water.

\subsection{Packing procedure of glass spheres of different size}

The following types and sizes of glass beads were used for mixtures: Beads with diameter 2, 3, and $4 \mathrm{~mm}$, were from Simax. Beads, code 4501, diameter $0.25-0.5 \mathrm{~mm}$ (average diameter $0.375 \mathrm{~mm}$ ) and code 4503 , diameter $0.75-1.0 \mathrm{~mm}$ (average diameter $0.875 \mathrm{~mm}$ ) were obtained from Sigmund Lindner. All suppliers stated in their technological information a density of $2500 \mathrm{~kg} / \mathrm{m}^{3}$ for the glass beads.

\subsubsection{Beads preparation}

Glass spheres of different size were first marked with inks of different colours. The following types of inks were used: Inks from Edding type T 100-2, T 100-3, T 100-4 and from Pebeo type 17, 21, 23, and 25 . The inks had good adhesion to glass and were non-soluble in the solution of glycerol. The beads were spread in a vessel with large bottom area, thus forming a thin layer, which makes easier the air-drying. Acetone-diluted ink was added to the vessel and periodic agitation avoided the agglomeration of the glass spheres (mainly in small sizes) during drying. The beads were sieved to remove the few existing agglomerates. A thin stable ink layer was formed on the surface of spheres, which were then used to build up the different particle mixtures.

\subsubsection{Mixing/packing procedure}

Dyed spheres were mixed in a certain mass proportion and put in a mixer. Mixer is formed by a horizontal cylindrical vessel containing inside a tri-helicoidal impeller with an external diameter very close to the inner wall of the vessel. The helicoids are coiled in different ways, which promotes the circulation of the spheres along the axis of the cylinder. The inclination of the helicoids promotes radial mixture. The impeller is driven by a motor with controlling rotation speed (Heidolph RZR 2041) (Fig. 1a). A solution of 90\% glycerol in water was then added to the spheres in a $15 \%$ mass proportion. Spheres and glycerol solution were mixed at $75 \mathrm{rpm}$ during $5 \mathrm{~min}$ inside the vessel. A very sticky mixture with sufficient adhesion between spheres was obtained.

The prepared mixture was transferred from the mixer to a square acrylic transparent column. The packing height varied in all experiments between 10

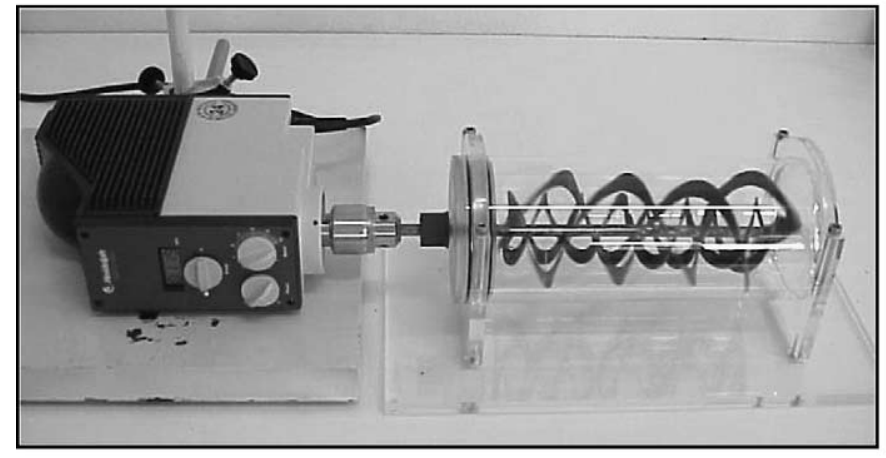

(a)

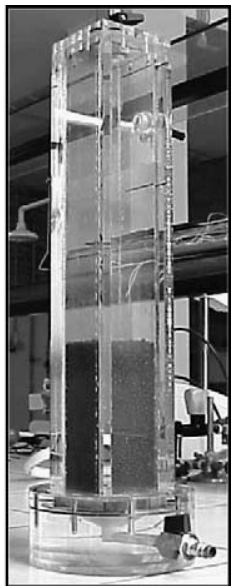

(b)

Fig. 1. Equipment used in the experiments to obtain mixture beds. (a) Mixing system. (b) General view of the square column with a dyed binary packing. 
and $15 \mathrm{~cm}$. The column was then gently filled with water, closed, and the glycerol was washed out with water entering the top of the column, at a high flow rate, during approximately $15 \mathrm{~min}$, until the glycerol concentration in the effluent was undetectable. Glycerol presence in the effluent was checked by refractometry. After dewatering the column, the mixture was tapped vertically until we got no variation on the packing thickness. Finally, the packing was completely dried with compressed air, which was stopped when no variation of the weight of column plus spheres was detected. For this purpose a balance from Precisa, model 30000D SCS with $d= \pm 0.1 \mathrm{~g}$, was used. Fig. 1b shows the column filled with a dyed binary packing. The column was square with $5 \mathrm{~cm}$ inner side by $40 \mathrm{~cm}$ height. The column faces were flat to avoid photographic distortion. A square wire cloth from Haver \& Boecker with $0.032 \mathrm{~mm}$ of aperture width was used to support the beads.

Images of the lateral sides of the packing were made with a digital camera from Sony, model MVC-FD91 with $\times 14$ optical zoom, to compare the particle fractions area displayed in the images with the fractional area effectively present in the mixture poured to the column. The images were treated by means of the software Image-Pro Plus from Media Cybernetics. First, a zone of the image was amplified to acquire with precision the colour of the different spheres. Then, the majority of the packing area was selected, in order to determine the percentage area correspondent to each spherical size.

After getting the images from the packing and after weighing the column plus spheres, the column was gently filled with water. Then, a high flow rate was used to remove all the entrapped air from the packing. The level of water inside the column was then fitted to the top surface of the packing and the weight of spheres plus water and column was measured. The weight of water present in the column in the zone underneath the layer support $(22.5 \mathrm{~g})$ was discounted after water porosity was calculated.

\subsubsection{Procedure for image treatment}

In a photographic image the particle mixture is represented in two dimensions. Therefore, if we have the image of spherical particles, the area occupied by one sphere (a disk) in the image is proportional to $A=$ $\pi(D / 2)^{2}$, where $D$ is the diameter of largest particles in the mixture. If we consider a certain mass $m_{D}$ of glass spheres with diameter $D$ and density $\rho_{g}$ in a mixture, the number of spheres $n_{D}$ present in that mass will be

$n_{D}=\frac{m_{D}}{\left(4 / 3 \pi(D / 2)^{3} \rho_{g}\right)}$

In the same way, for smallest glass spheres of diameter $d$

$n_{D}=\frac{m_{D}}{\left(4 / 3 \pi(d / 2)^{3} \rho_{g}\right)}$.

The total area corresponding to the spheres of diameter $D$ is

$A_{D}=n_{D} \pi\left(\frac{D}{2}\right)^{2}=\frac{m_{D}}{\left(4 / 3 \pi(d / 2) \rho_{g}\right)}$.

The total area corresponding to the spheres of diameter $d$ is, respectively

$A_{d}=\frac{m_{D}}{\left(4 / 3 \pi(d / 2) \rho_{g}\right)}$

The percentage area of spheres of diameter $D$ in the mixture will then be:

$A_{D}(\%)=\frac{A_{D}}{A_{d}+A_{D}} 100=\frac{m_{D} / D}{\left(m_{D} / D\right)+\left(m_{d} / d\right)} 100$

The total mass of the mixture is $m_{T}=m_{D}+m_{d}$. If $x_{D}$ and $x_{d}$ are the mass fractions of largest and smallest particles in the mixture then

$A_{D}(\%)=\frac{x_{D} / D}{\left(x_{D} / D\right)+\left(x_{d} / d\right)} 100$

In general, for a mixture of $\mathrm{n}$ sizes of spheres: $A_{i}=$ $\left(x_{i} / d_{i}\right) / \sum_{i=1}^{n}\left(x_{i} / d_{i}\right)$.

Finally, for instance, in the case of a binary mixture, uniform distribution of particles fractions within the packing space would correspond in the taken image to the percentage area occupied by each fraction of dyed particles with respect to Eq. (7). In Fig. 2 we show an example of the obtained images and the calculated area occupied by each fraction recurring to Image-Pro Plus. The expected results predicted by Eq. (7) should be $A_{D}=11.1 \%$. The result obtained by means of image analysis was $11.9 \%$. This indicates that we have obtained a good mixture in terms of particle fractions distribution in the mixture volume. Even for ternary 


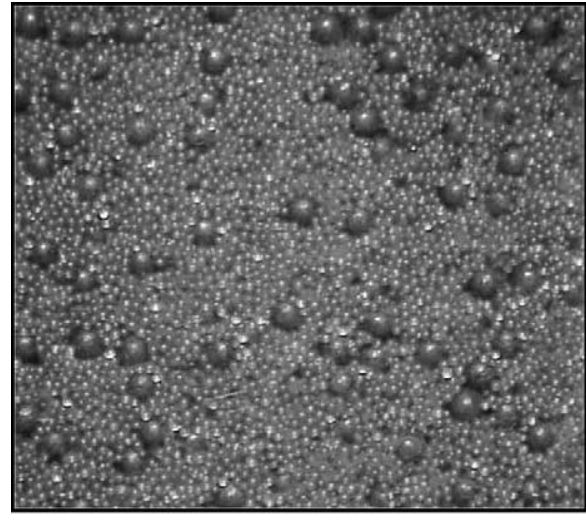

(a)

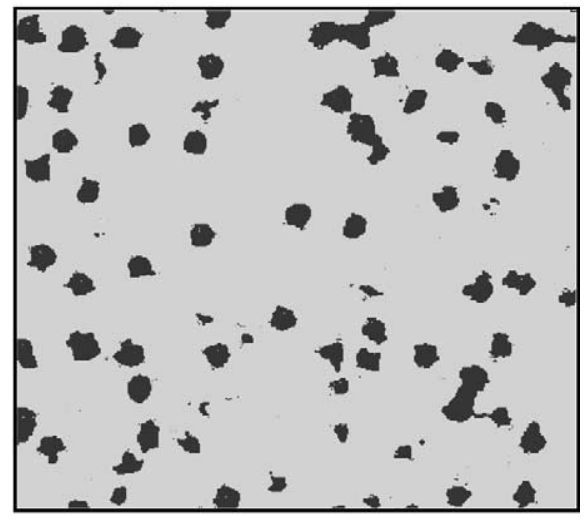

(b)

Fig. 2. Photo (a) and treated image (b), of a mixture treated by Image-Pro Plus. The mixture contains $30 \%$ of $3 \mathrm{~mm}$ spheres (black) and $70 \%, 0.875 \mathrm{~mm}$ spheres (grey).

mixtures, which are outside of the framework of the present discussion, the difference of $A_{D}$ between calculated and determined by image analysis was less than $\pm 10 \%$.

\subsubsection{Procedure to check the uniformity of distribution}

Each captured image was divided in eight vertical sectors. The dark glass beads present in each sector were counted. A chi-square fitness test was made to check whether the distribution could be considered as uniform. If there is no wall effect, then there will be no accumulation of beads at the picture lateral borders, corresponding to the vessel edges.

On the other hand, three pictures of each packing were taken for every four faces of the vessel. The average and the standard deviation for each colour fraction were calculated.

\section{Results and discussion}

\subsection{Distirbution}

In all cases, the application of the chi-square fitness test showed that the hypothesis of a uniform distribution must be accepted at the $1 \%$ significance level. This means that there was no evidence of a wall effect, which agrees with the experimental results from Al-Dahhan et al. [14].
For 27 different packings of binary mixtures and for 18 packings of ternary mixtures the standard deviation obtained for each face varied between 0.12 and $1.42 \%$. The mean standard deviation obtained was $0.89 \%$. Therefore we may say that the results obtained are reproducible and that no significant variation occurs in the captured image distributions of the four faces.

\subsection{Complete mixtures}

As was mentioned above, numerous models of particle mixed beds are built on the basis of averaged parameters when physical characteristics are defined with respect to the overall space occupied by mixture (Fig. 3), where boxes schematically represent the overall mixture space: (a) mixture enriched by small particles; (b) mixture nearby the minimum porosity (maximum packing density); (c) mixture enriched with large particles. In case (b), distribution of large particles is close to the continuous skeleton structure but, due to the small particle size ratio, fine particles are inserted in the skeleton. In case (c), small particles dilute the skeleton of large particles in the packing. We name this type of mixtures complete mixtures, where all particles are distributed evenly within the mixture volume.

Fig. 4 represents the images of two adjacent faces of the same packing, corresponding to region " $b$ " from Fig. 3b (minimum porosity). The predicted 


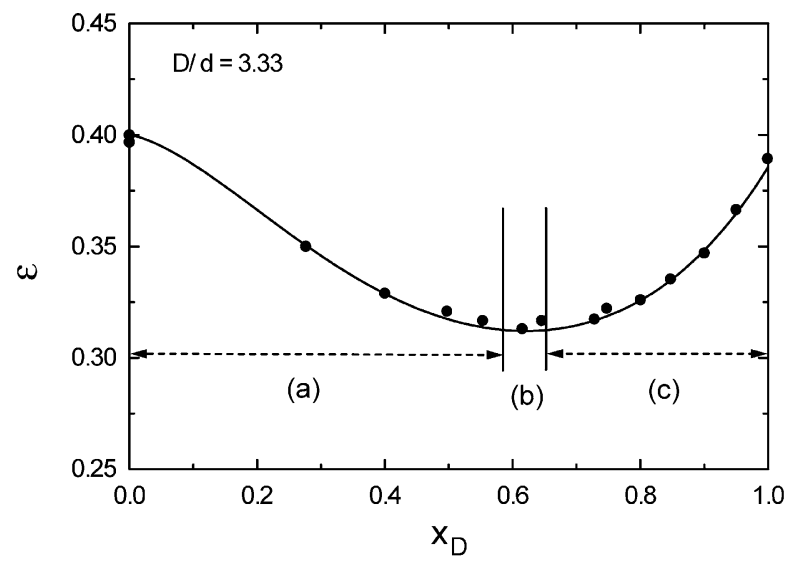

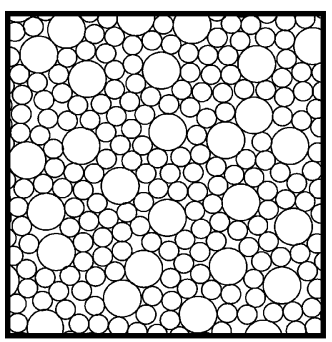

(a)

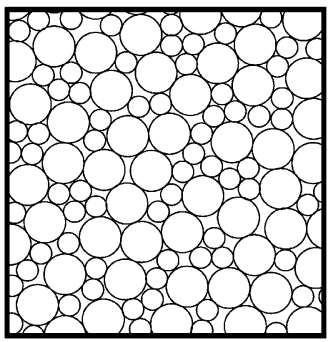

(b)

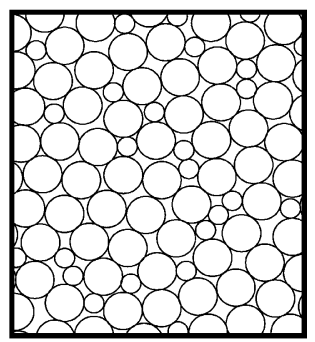

(c)

Fig. 3. Binary packing - dependence of the mixture porosity $\varepsilon$ on volume fraction of large particles $x_{D}$ for particle size ratio $D / d=3.33$ : points-experimental, curve-model [8]. Boxes schematically represent zones in the mixture space. (a) mixture enriched with small particles; (b) mixture close to the minimum porosity (maximum packing density); (c) mixture enriched with large particles.

result by Eq. (7) is $A_{D}=24.7 \%$. The obtained results by Image-Pro Plus were $23.4 \%$ (Fig. 4 a) and $25.6 \%$ (Fig. 4b), with an average of $24.5 \%$-less than $1 \%$ deviation.
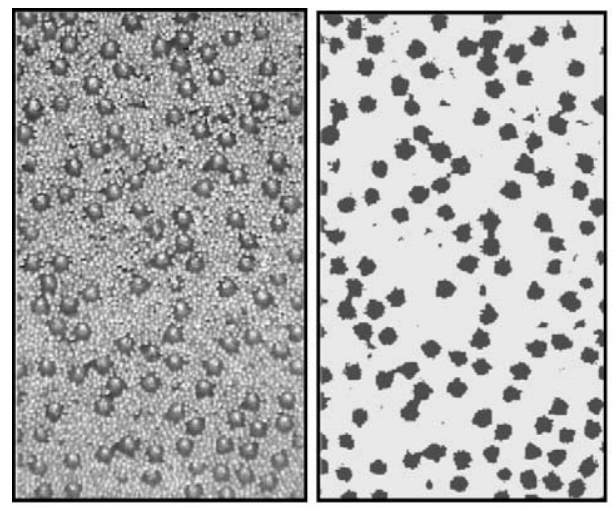

(a)
An experiment performed in the region of minimum porosity is given below. Complete packing appears in Fig. 5 and the image analysis gave a result of $A_{D}=40.2 \%$, which is quite close-a deviation of
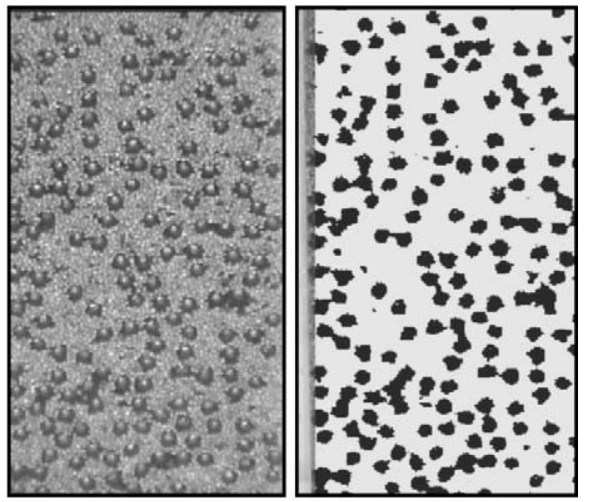

(b)

Fig. 4. Two faces of the same packing (a) and (b), in the region of minimum porosity. The packing contains $60 \%$ of $4 \mathrm{~mm}$ spheres (black) and $40 \%, 0.875 \mathrm{~mm}$ spheres (grey). Right-hand rectangles in each sector are treated as images. 


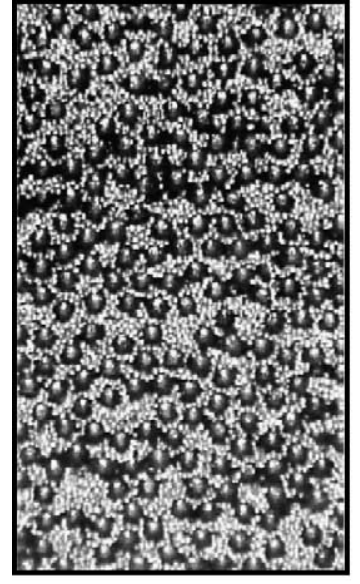

(a)

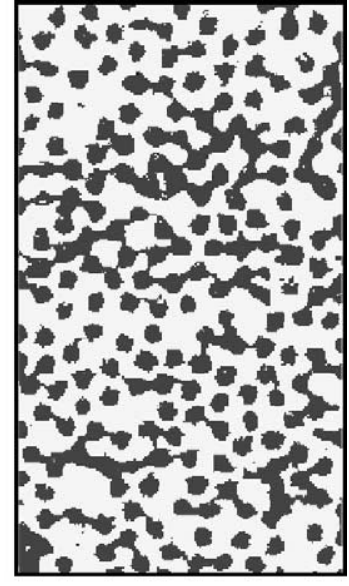

(b)
Fig. 5. Photo image (a) and treated image (b) of a packing obtained in the region $c$ from Fig. 3. The mixture contained $75 \%$ of $4 \mathrm{~mm}$ spheres (black) and 25\%, $0.875 \mathrm{~mm}$ spheres (grey).
$1.5 \%$ - to the expected result of $39.6 \%$ obtained by Eq. (7).

\subsection{Complex mixtures}

When the particle size ratio is below 0.41 (cube packing) -0.13 (hexagonal pacing), small particles are able to intrude in between the large particles skeleton and cause a segregation effect in the gravity field when the volume fraction of large particles exceeds the minimum porosity threshold (Fig. 6). The segregation effect results in the separation of the mixture in two layers-the layer of the complete mixture at the bottom with porosity around minimum packing porosity (Fig. 6, c1) and the top layer of pure large particles packing presumably freed of small particles. We named this type of mixture enriched with large particles as a complex non-homogeneous mixture.

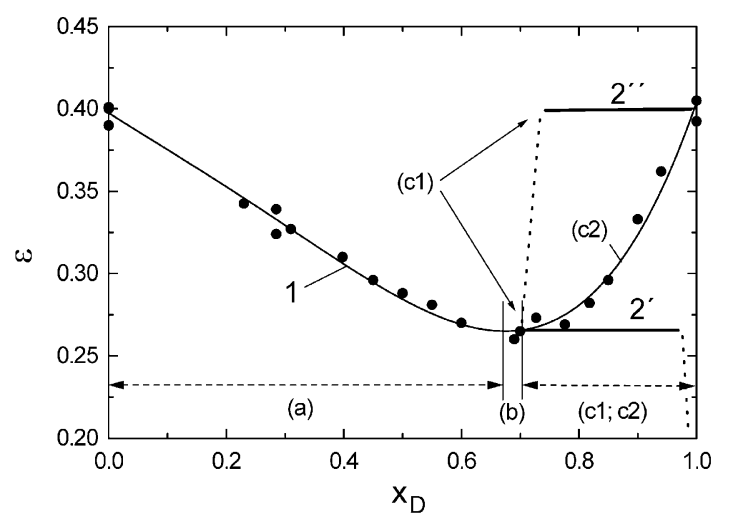

(a)

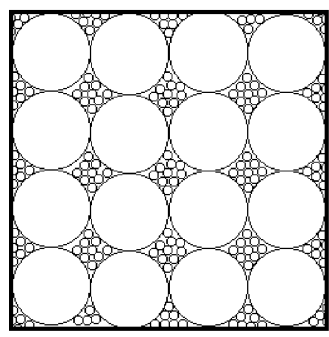

(b)

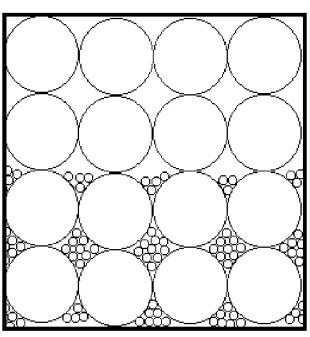

(c1)

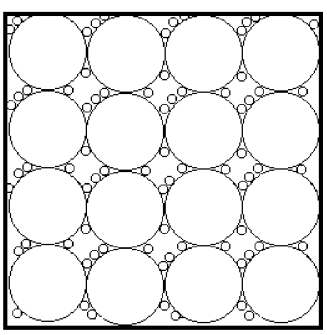

(c2)

Fig. 6. (a) Dependence of the mixture porosity $\varepsilon$ on the volume fraction of large particles $x_{D}$ for particle size ratio $D / d=10.7$ when the segregation effect takes place: points-experimental, curve 1 -fitting function. Lines $2^{\prime}$ and $2^{\prime \prime}$ correspond to the porosity of pure large particles packing and mixed layer with porosity close to the minimum packing porosity. Dotted lines-transition zones. Boxes schematically represent the overall mixture arrangement: (b) mixture close to the minimum porosity (maximum packing density); (c1) segregated (complex) mixture enriched with large particles; (c2) mixture enriched with large particles when small particles are distributed regularly within the skeleton. 


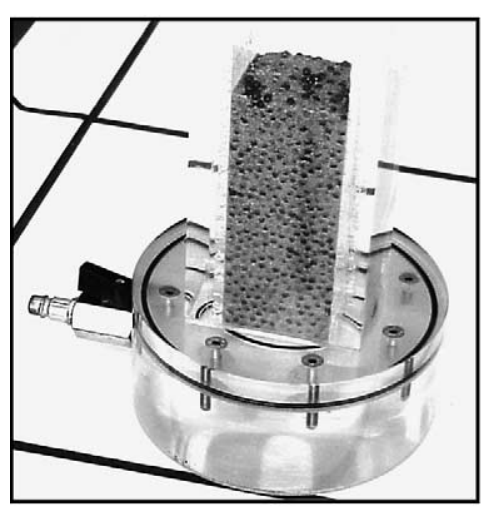

(a)
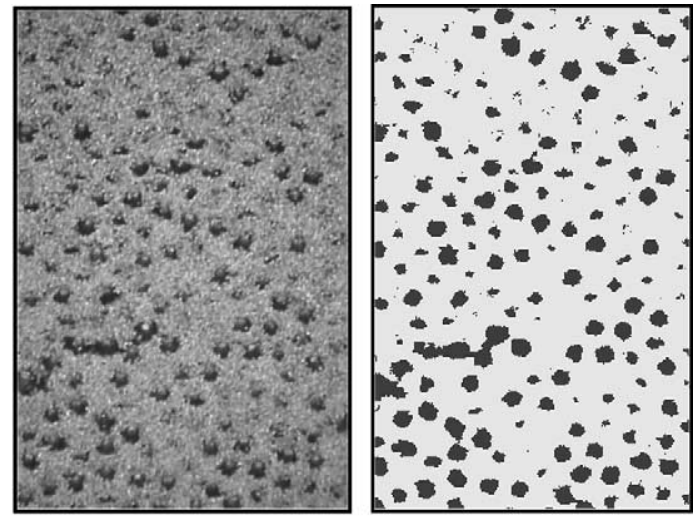

(b)

Fig. 7. Packing in the region of minimum porosity (a) and images (b) of the mixture with $70 \% 4 \mathrm{~mm}$ (black), $30 \% 0.375 \mathrm{~mm}$ spheres (grey).

Each layer has a porosity close to the porosity of pure large particles packing $\left(\approx \varepsilon_{D}^{0}\right)$ and porosity corresponds to the maximum particles packing or minimum porosity $\left(\varepsilon_{\min }\right)$, whereas the average porosity corresponds to curve (c2) when it is assumed that all small particles are regularly distributed within the skeleton void of large particles.

The assumption of packing as a complete mixture in the region $(\mathrm{c} 1 ; \mathrm{c} 2)$ is valid if the physical processes (fluid flow, diffusion, etc.) follow the additive law [5].
In some cases the spatial solid distribution plays a major role in processes such as filtration, diffusion of large molecules when they are in scale with packing particles, flow of rheologically complex fluid, etc. Also, a characteristic such as the tortuosity strongly depends on spatial particles arrangement [52] and must be taken into consideration.

An example of complete packing in the region of minimum porosity is given in Fig. 7. The percentage area of the large component is $A_{D}=16.89 \%$. So, we may conclude that we have obtained a good

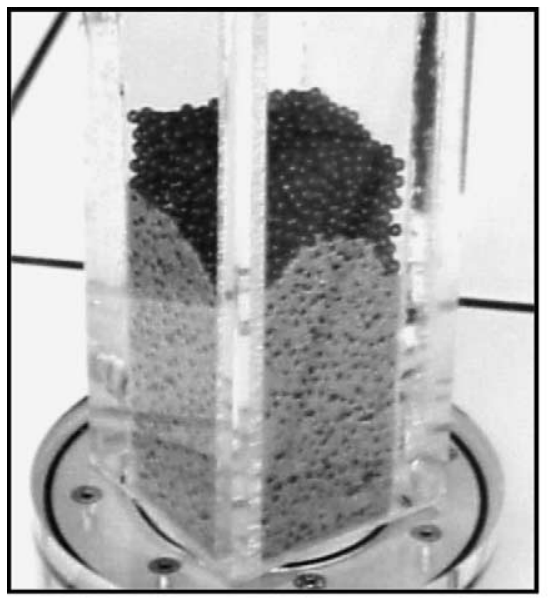

(a)
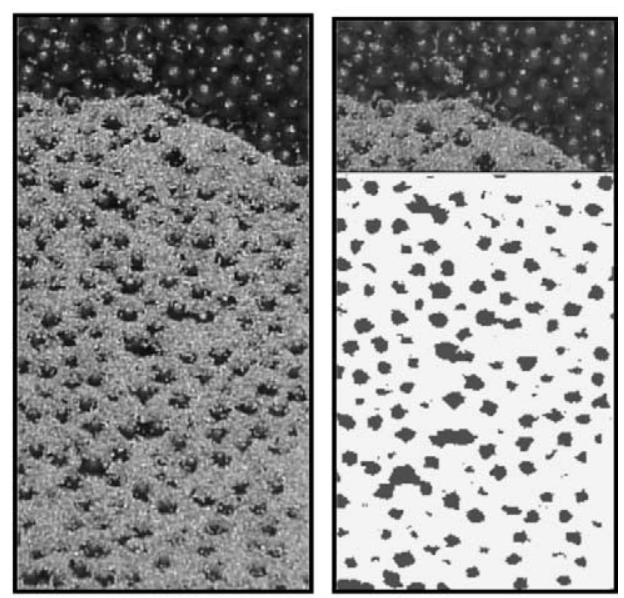

(b)

Fig. 8. Complex packing (a) and image (b) of percentage area occupied by each fraction, recurring to Image-Pro Plus, for the experiment in the region $c 1$ (Fig. 6). The mixture contains $75 \%, 4 \mathrm{~mm}$ spheres (black) and 25\%, $0.375 \mathrm{~mm}$ spheres (grey). The packing staying below the segregation zone corresponds to the complete packing at the minimum porosity region. 

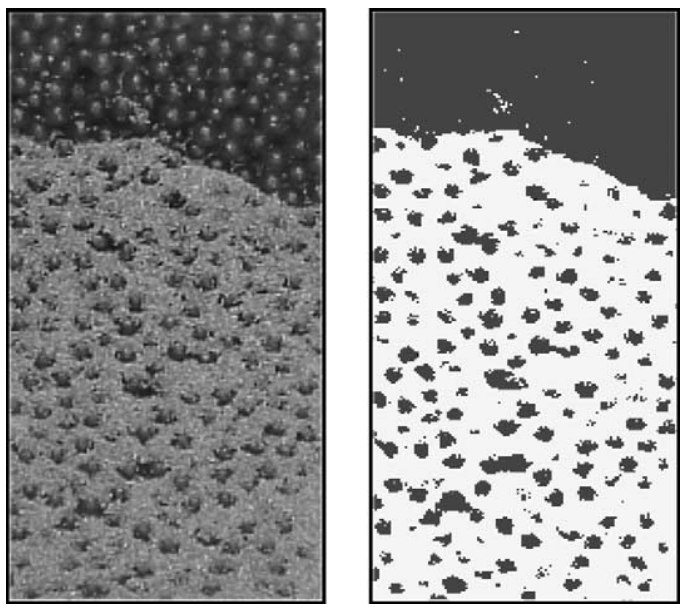

Fig. 9. Photo and treated image of the packing with composition $75 \%, 4 \mathrm{~mm}$ spheres (black) and 25\%, $0.375 \mathrm{~mm}$ spheres (grey) when the packing is considered as a whole (both layers).

mixture since the theoretical percentage of area for $4 \mathrm{~mm}$ should be $17.95 \%$ by Eq. (7).

In turn, for the complex binary mixture enriched with large particles, after the tapping procedure, we can clearly see the segregation effect in Fig. 8.

By analysis with Image-Pro Plus we can conclude that the zone where the mixture is stable is composed of $70 \% 4 \mathrm{~mm}$ beads and $30 \% 0.375 \mathrm{~mm}$ beads. The measured $A_{D}$ was $17.37 \%$, whereas the value predicted by Eq. (7) is equal to $17.95 \%$, showing the validity of our previous assumptions. Therefore, for the complex mixture the layer model can be applied [55], for the calculation of tortuosity, permeability, diffusivity, etc, and the bottom layer represents the complete mixture of nearby minimum porosity and the top layer is the pure large particles packing.

The last result, considering all the packing, is an example of a bad mixture between the two components. Analysing all the packing by Image-Pro Plus (Fig. 9) we obtain $A_{D}=34.8 \%$ which is far from the predicted result by Eq. (7) of $22 \%$. We may conclude that any important heterogeneity in terms of mixture in the porous media (even in the interior) will affect the amount of the components available on the faces of the packing, giving rise to a deviation of Eq. (7).

\section{Conclusion}

The mixing method with glycerol proved to be effective as was confirmed by image analysis. The deviations between the results obtained by image analysis and the expected results were in general small. It is thus possible to use this mixing method to obtain a wide range of binary of mixtures.

The image analysis of coloured mixtures gave promising results in ternary mixtures. However, in the case of ternary mixtures, the coexisting three different bead sizes increase the number of miscalculations to the increasing number of shaded areas. The obtained results will be further improved when the software currently under development will be able to remove automatically the shadows cast by the spheres.

As a secondary but still important result, it was shown that segregated layer mixtures give rise to bottom layers with the minimum porosity.

\section{Acknowledgements}

The authors wish to thank Fundação para a Ciência e Tecnologia (FCT) for having provided the funds to perform this work through the project POCTI/EQU/37500/2001, as well as for the grant accorded to A.Yelshin. This project was partially funded by FEDER.

\section{References}

[1] J.A. Currie, Brit. J. Appl. Phys. 11 (1960) 318-324.

[2] C.N. Satterfield, Mass Transfer in Heterogeneous Catalysis, MIT Press, Cambridge, 1970.

[3] J. Bear, Dynamics of Fluids in Porous Media, Elsevier, Amsterdam, 1972.

[4] J. Bear, Y. Bachmat, in: J. Bear, M.Y. Corapcioglu (Eds.), Fundamentals of Transport Phenomena in Porous Media, NATO ASI Series E: Applied Sciences, vol. 82, Nijhoff Publishers, Martinus, The Netherlands,1984, pp. 3-61.

[5] R.H. Perry, D.W. Green, J.O. Maloney (Eds.), Perry's Chemical Engineers Handbook, McGrow-Hill, 1988, p. 5-54.

[6] P.M. Adler, Porous Media: Geometry and Transports, Butterworth-Heinemann, Stoneham U.S.A., 1992.

[7] S.V. Tsirel, Int. J. Rock Mech. Min. Sci. 34 (1997) 263-273.

[8] M. Mota, J.A. Teixeira, A. Yelshin, Trans. Filtrat. Soc. 1 (2001) 101-106.

[9] J.-P. Latham, A. Munjiza, Y. Lu, Powder Technol. 125 (2002) $10-27$. 
[10] S. Liu, Z. Ha, Powder Technol. 126 (2002) 283-296.

[11] M. Mota, J.A. Teixeira, W.R. Bowen, A. Yelshin, Miner. Eng., 16,2003 , in press.

[12] M.J. MacDonald, C.-F. Chu, P.P. Pierre, K.M. Ng, AIChE J. 37 (1991) 1583-1588.

[13] C. Wightman, P.R. Mort, F.J. Muzzio, R.E. Riman, E.K. Gleason, Powder Technol. 84 (1995) 231-240.

[14] M.H. Al-Dahhan, Y. Wu, M. P: Dudukovic, Ind. Eng. Chem. Res. 34 (1995) 741-747.

[15] R. P: Zou, A.B. Yu, Powder Technol. 88 (1996) 71-79.

[16] M. Shahinpoor, Powder Technol. 25 (1980) 163-176.

[17] M. Suzuki, K. Makino, M. Yamada, K. Iinoya, Int. Chem. Eng. 21 (1981) 482-488.

[18] R.A. Greenkorn, AIChE J. 27 (1981) 529-543.

[19] D.G. Huizenga, D.M. Smith, AIChE J. 32 (1986) 1-6.

[20] T. Wright, D.M. Smith, D.L. Stermer, Ind. Eng. Chem. Res. 26 (1987) 1227-1232.

[21] G. Liu, K.E. Thompson, Powder Technol. 113 (2000) 185196.

[22] A.R. Gupte, Chem. Ing. Techn. 43 (1971) 754-761.

[23] A.B. Yu, N. Standish, Ind. Eng. Chem. Res. 30 (1991) 1372 1385.

[24] A.B. Yu, N. Standish, Powder Technol. 76 (1993) 113-124.

[25] A.B. Yu, N. Standish, L. Lu, Powder Technol. 82 (1995) 177-189.

[26] M. Mota, J.A. Teixeira, R. Bowen, A. Yelshin, in: Proceedings of 8th World Filtration Congress, 3-7 April 2000, vol. 1, Filtration Society, Brighton, UK, 2000, pp. 57-60.

[27] M. Mota, J.A. Teixeira, A. Yelshin, Sep. Purif. Technol. 15 (1999) 59-68.

[28] R. Venugopalan, D. Sathiyamoorthy, H.S. Gadiyar, Powder Technol. 114 (2001) 229-236.

[29] N. Ouchiyama, T. Tanaka, Ind. Eng. Chem. Fundam. 20 (1981) 66-71.

[30] R.K. Sharma, D.L. Cresswell, E.J. Newson, Ind. Eng. Chem. Res. 30 (1991) 1428-1433.

[31] R. Aris, The Mathematical Theory of Diffusion and Reaction in Permeable Catalysts, vol. 1, Clarendon Press, Oxford, 1975.

[32] C.N. Satterfield, Heterogeneous Catalysis in Practice, McGraw-Hill, 1980.

[33] C.J. Geankopolis, Transport Processes and Unit Operations, Allyn and Bacon, U.S.A., 1983.

[34] J.R. Welty, C.E. Wicks, R.E. Wilson, Fundamentals of Momentum, Heat, and Mass Transfer, Wiley, Singapore, 1984, pp. $477-498$.
[35] D.E. Rosner, Transport Processes in Chemically Reacting Flow Systems, Butterworths, U.S.A., 1986.

[36] N.E. Olague, D.M. Smith, M. Ciftcioglu, AIChE J. 34 (1988) 1907-1909.

[37] S.M. Walas, Chemical Reaction Engineering Handbook of Solved Problems, Gordon \& Breach, Amsterdam, 1995, pp. 721-722.

[38] H.W. Blanch, D.S. Clark, Biochemical Engineering, Marcel Dekker, New York, 1996.

[39] M. Mota, J.A. Teixeira, A. Yelshin, in: Feyo de Azevedo, E. Ferreira, K. Luben, P. Osseweijer (Eds.), Proceedings of 2nd European Symposium on Biochemical Engineering Science, University of Porto, Porto, 16-19 September, 1998, pp. 93-98.

[40] A.D. Rosato, D. Yacoub, Powder Technol. 109 (2000) 255261.

[41] J.L. Turner, M. Nakagawa, Powder Technol. 113 (2000) 119123.

[42] E. Caglioti, V. Loreto, H.J. Herrmann, M. Nicodemi, Phys. Rev. Lett. 79 (1997) 1575-1578.

[43] M. Nicodemi, Physica A 257 (1998) 448-453.

[44] M. Nicodemi, A. Coniglio, H.J. Herrmann, Phys. Rev. E 55 (1997) 3962-3969.

[45] E. Caglioti, A. Coniglio, H.J. Herrmann, V. Loreto, M. Nicodemi, Physica A 257 (1998) 419-423.

[46] M. Suzuki, T. Oshima, Powder Technol. 43 (1985) 147153.

[47] N. Ouchiyama, T. Tanaka, Ind. Eng. Chem. Fundam. 23 (1984) 490-493.

[48] J.V. Milewski, Ind. Eng. Chem. Prod. Res. Dev. 17 (1978) 363-366.

[49] E. Abe, H. Hirosue, J. Chem. Eng. Jpn. 15 (1982) 490-493.

[50] A.B. Yu, N. Standish, A. McLean, J. Am. Ceram. Soc. 76 (1993) 2813-2816.

[51] A.B. Yu, R.P. Zou, N. Standish, Ind. Eng. Chem. Res. 35 (1996) 3730-3741.

[52] A. Yelshin, M. Mota, J. Teixeira, in: Proceedings of Int. Conference Filtech Europa-97, Düsseldorf, Filtration Society, Horsham (UK), 14-16 October, 1997, pp. 327-334.

[53] M.F. Mathias, G.P. Muldowney, Chem. Eng. Sci. 55 (2000) 4981-4991.

[54] P.J.T. Mills, J.P.K. Seville, P.C. Knight, M.J. Adams, Powder Technol. 113 (2000) 140-147.

[55] M. Mota, J.A. Teixeira, A. Yelshin, Biotechnol. Progr. 18 (2002) 807-814. 